\title{
Predictable Risk Factors of Upper-Extremity Deep Venous Thrombosis in a Level I Trauma Center
}

\author{
Scarlett Tohme $\mathbb{D}^{1}$ \\ Aparna Vancheswaran' \\ Kyle Mobbs' \\ Jessica Kydd ${ }^{2}$ \\ Nisha Lakhi ${ }^{1,2}$ \\ 'New York Medical College, School of \\ Medicine, Department of Surgery, \\ New York, NY, USA; ${ }^{2}$ Richmond \\ University, Medical Center, Department \\ of Trauma Surgery, Staten Island, \\ NY, USA
}

\begin{abstract}
Background: Venous thromboembolism is a common cause of morbidity and mortality in hospital patients, especially that of the lower extremities. Risk factors and diagnostic elements of upper-extremity deep-vein thrombosis (UEDVT) are poorly understood compared to those of the lower extremities. The primary objectives of this study were to identify predictive risk factors of secondary UEDVT.
\end{abstract}

Methods: This retrospective study included all nonpregnant patients aged $>18$ years who had undergone upper-extremity duplex scans to check for the presence of secondary UEDVT at Richmond University Medical Center from January 2014 to March 2020. Patients were stratified by presence or absence of UEDVT. Collected data points included patient demographics, comorbidities, central-line use, platelet count at time of scan, length of stay, and overall mortality. IBM 27.0 was used for all statistical analysis, with $\mathrm{p}<0.05$ considered significant.

Results: A total of 1,009 upper extremity venous duplex studies were included. There were no significant differences in age, sex, race, or mean platelet levels between patients diagnosed with DVT and those without $(p<0.05)$. After multinomial regression analysis, central venous catheter (CVC; $26.8 \%$ versus $78.5 \%$, aOR $1.770,95 \%$ CI $1.150-2.725 ; p<0.002$ ), peripherally inserted central catheter (PICC) line (17.5\% versus $82.5 \%$, aOR3.254, 95\% CI 1.997-5.304; $p<0.001)$, hypertension $(67.8 \%$ versus $28.8 \%$, aOR 1.641 , 95\% CI 1.136 $2.369 ; p<0.001)$, chronic kidney disease (CKD; 34.5\% versus $65.5 \%$, aOR $1.743,95 \% \mathrm{CI}$ $1.201-2.531 ; p<0.001)$, and malignancy $(27.1 \%$ versus $74.6 \%$, aOR $1.475,95 \%$ CI 0.994 $2.190 ; p<0.053)$ were found to be independent predictors of UEDVT.

Conclusion: Use of CVC or PICC line, preexisting diagnosis of hypertension, malignancy, and CKD were independent risk factors of UEDVT, while there was no significant correlation between increased platelet levels and UEDVT.

Keywords: deep-vein thrombosis, upper extremity, risk factors, thromboembolism, thrombocytosis

\section{Plain-Language Summary}

The presence of central venous catheters, peripherally inserted central catheter lines, and morbidities of hypertension, chronic kidney disease, and malignancy were shown to be independent risk factors of the development of upper extremity deep-vein thrombosis in this retrospective study of 1,009 upper-extremity Doppler scans at a level I trauma center. This information will aid health-care providers to more accurately identify patients at risk of upper extremity deep-vein thrombosis and guide subsequent management.
Correspondence: Scarlett Tohme New York Medical College, School of Medicine, Department of Surgery, 40 Sunshine Cottage Road, Valhalla, NY, 10595, USA,

Tel + I 347-277-3993

Email stohme@student.nymc.edu 


\section{Introduction}

Venous thromboembolism is a major public-health problem and one of the leading causes of morbidity and mortality in hospitalized patients. ${ }^{1,-3}$ Although there is vast information and evidence describing the risk factors and morbidity and mortality outcomes in lower-extremity deep-vein thrombosis (LEDVT), less is known about venous thrombosis of the upper extremity. There are two forms of upper-extremity DVT (UEDVT): primary and secondary. ${ }^{1}$ The primary form is known as PagetSchrötter syndrome, and occurs in the dominant arm of younger athletic patients involved in excessive and repetitive motion of the upper extremities. ${ }^{1}$ The secondary form occurs most commonly in patients with central venous catheter (CVC) use or those with malignancy. ${ }^{1}$ This latter form of UEDVT has seen increased incidence over the past decade, with the rate of UEDVT in patients with central catheterization ranging between $14 \%$ and $23 \%{ }^{1}$ Higher morbidity and mortality rates have been associated with UEDVT than LEDVT, suggesting that a diagnosis of UEDVT may be a predictor of morbidity and mortality. ${ }^{1,2}$

A majority of upper-extremity venous thrombosisrelated diseases and conditions described in the literature are associated with peripherally inserted central catheter (PICC lines) lines, indwelling CVCs, immobilization $>3$ days, and malignancy. ${ }^{3}$ Other risk factors include severe kidney failure, inflammatory diseases, and deficiency of anticoagulant factors. ${ }^{1-5}$ Although it is becoming clearer that the aforementioned risk factors play an important role in the incidence and outcomes of UEDVTs, analyses on the effects of hereditary or acquired biological thrombophilia and the impact on reactive thrombocytosis, defined as a consistent platelet count $\geq 450 \times 10^{9} / \mathrm{L}$, are lacking. ${ }^{6,7}$

The primary objective of this study was to identify predictive risk factors of secondary UEDVT in patients presenting to a level I trauma center. This study will fill knowledge gaps by providing a comprehensive evaluation of demographic factors, disease comorbidities, and hematologic indices in terms of their contribution to of UEDVT incidence. We also compare independent risks associated with various types of invasive venous catheters. A comprehensive understanding of these risk factors can aid health-care professionals in identifying patients at risk of developing an UEDVT and possibly mitigate the morbidity and mortality associated with this condition.

\section{Methods}

This IRB-approved $(14,231)$ retrospective-cohort study was conducted at Richmond University Medical Center, a level I trauma center in Staten Island, NY between January 2014 and March 2020. Informed consent was waived by the IRB, due to the retrospective nature of the study. All patient data were kept secure as per the relevant data-protection and privacy regulations. This study was conducted in accordance with the Declaration of Helsinki. The medical records of 1,324 patients that had had an upper-extremity duplex scan during the study period were identified using CPT codes. The patient population in this study included any adult patient who had undergone a venous duplex Doppler scan of the upper extremities who presented with any upper-extremity symptoms, whether of a unilateral upper extremity or of the bilateral upper extremities. Patients who had undergone venous duplex Doppler scans of the upper extremity were excluded from the study if they were aged $<18$ years, if they were diagnosed with primary upper extremity DVT, (Paget-Schrötter syndrome, pregnant at the time of the diagnostic exam, had been diagnosed with upperextremity superficial venous thromboses, or requiring a scan but had not presented with any symptoms of the upper extremity and/or if the venous duplex study was ordered for arteriovenous fistula mapping or monitoring. By definition, only thromboses of the radial, ulnar, axillary, brachial, and subclavian veins were considered for a diagnosis of UEDVT.

Cases were divided into sub-cohorts based on the presence or absence of UEDVT on duplex scan to allow for comparative analyses. Collected demographic data points included, age, sex, race, and inpatient/outpatient status. Presence of comorbidities was identified using ICD codes, and included hypertension, heart disease (congestive heart disease, history of myocardial infarction, coronary artery disease), diabetes mellitus, thrombophilia, pulmonary disease (asthma, chronic obstructive pulmonary disease), malignancy, chronic kidney disease (CKD), history of implanted cardiac implantable electronic devices or implanted pacemakers, use or placement of CVCs and/or PICC lines during current hospitalization, platelet count at time of duplex scan, length of inpatient hospital stay, and mortality. Presenting diagnosis and indication for upper-extremity duplex scan were recorded. 


\section{Statistical Analysis}

Statistical analysis was carried out using SPSS 27.0. Univariate analyses for continuous variables were compared using Student's $t$-test or Mann-Whitney $U$ test. Categorical data were compared using $\chi^{2}$ test or Fisher's exact tests. $P<0.05$ was considered statistically significant. Variables that were statistically significant on univariate analysis were tested for interaction of terms. Nonredundant variables were entered into a multivariate logistic regression model to test for adjusted associations. Regression imputation was used to preserve all cases and replace missing data with a probable value estimated by other available information. Variables that retained statistical significance are reported as adjusted aOR with 95\% CIs.

\section{Results}

A total of 1,329 reports were initially screened for venous duplex scans based on CPT codes. Four studies were excluded because of patients $<18$ years of age, five for pregnancies, ten for cancellation of duplex scans, 18 for diagnoses of upper-extremity superficial venous thromboses, and 283 for primary indication of arteriovenous fistula mapping or monitoring. In total, 1,009 venous duplex scans of the upper extremity were included in the analyses, where $445(44.1 \%)$ were of the right upper extremity, $541(53.6 \%)$ of the left upper extremity, and $23(2.3 \%)$ of bilateral upper extremities. Indications for these upper-extremity venous duplex studies included upper-extremity swelling (349), pain (125), pain and swelling (435), or suspicion of UEDVT secondary to known risk factors of LEDVTs as previously mentioned, in addition to symptoms of pain and/or swelling (100). Of the 1,009 encounters, $177(17.5 \%)$ were found to have a diagnosis of UEDVT, of which $73(41.2 \%)$ were of right upper extremities, 94 (53.1\%) of left upper extremities, and ten $(5.6 \%)$ of bilateral upper extremities (Table 1). All cases followed the American Society of Hematology guidelines for DVT prophylaxis for hospitalized patients that were contemporary with the date of occurrence.

\section{Patient Demographics and Comorbidities}

Mean age of patients diagnosed with UEDVT was 65.44 \pm 15.75 years. There was no difference in demographic factors (age, race, or sex) between those diagnosed with UEDVT and those without (Table 1). Of patients investigated for UEDVT, 555 (49.1\%) were female and 454
(51.9\%) male. In terms of race of patients investigated for UEDVT 496 (44.1\%) were Caucasian, 281 (32.2\%) African American, 163 (16.9\%) Hispanic or Latino, and $69(6.8 \%)$ other. Race was not significantly correlated with diagnosis of UEDVT. (Table 1). Overall, 675 (66.9\%) were inpatients and 334 (33.1\%) outpatients. Inpatients all followed the standard hospital inpatient anticoagulation protocol.

Of the comorbidities studied, pacemaker/implantable cardioverter defibrillator presence, diagnosis of diabetes, heart disease, thrombophilia, and pulmonary disease were not found to be associated with significantly increased risk of UEDVT ( $p>0.05$, see Table 1). Inpatient versus outpatient status ( $81.4 \%$ of inpatients diagnosed with UEDVT versus $18.6 \%$ of outpatients diagnosed with UEDVT), hypertension $(67.8 \%$ with hypertension diagnosed with UEDVT versus $28.8 \%$ without hypertension diagnosed with UEDVT), CKD (34.5\% with CKD diagnosed with UEDVT versus $65.5 \%$ without CKD diagnosed with UEDVT), CVC use $(21.5 \%$ with CVC use diagnosed with UEDVT versus $87.5 \%$ of no CVC use diagnosed with UEDVT), PICC-line use (17.5\% with PICC-line use diagnosed with UEDVT versus $82.5 \%$ with no PICC-line use diagnosed with UEDVT), and malignancy (27.1\% with malignancy diagnosed with UEDVT vs $74.6 \%$ without malignancy diagnosed with UEDVT) were found to be significantly correlated with diagnosis of UEDVT $(p<0.05$, see Table 2).

\section{Clinical Factors}

Of the 803 patients that had hematological test results, mean platelet levels at time of ultrasound for patients diagnosed with UEDVT was $256.76 \pm 128.48$ and those without the diagnosis $261.69 \pm 132.64$. This difference was not significant $(p=0.714)$, and distribution of platelet levels across individuals with UEDVT closely resembled that of individuals without UEDVT (Figure 1). Of 675 inpatient encounters, mean length of stay was similar for patients diagnosed with UEDVT $21.37 \pm 21.31$ days and patients without UEDVT $18.57 \pm 25.16)$ days $(p=0.225)$.

\section{Multivariate Logistic Regression Analysis}

Multivariate logistic regression analysis was performed to assess independent predictors of UEDVT. The results showed that the model containing six variables outperformed the null model $\left(\chi^{2}=52.530, d f 5 ; p<0\right)$. Pearson and deviance $\chi^{2}$ tests for goodness of fit weresignificant $(p>0.05)$, indicating that the model was a good fit for our data (Pearson $\chi^{2}=19.892$ 
Table I Demographic characteristics and comorbidities of cases

\begin{tabular}{|c|c|c|c|}
\hline $\begin{array}{l}\text { Characteristicl } \\
\text { Comorbidity }\end{array}$ & DVT, n (\%) & $\begin{array}{c}\text { No DVT, } \\
\text { n (\%) }\end{array}$ & Significance \\
\hline $\begin{array}{l}\text { Sex } \\
\text { Female } \\
\text { Male }\end{array}$ & $\begin{array}{l}87(49.2 \%) \\
90(50.8 \%)\end{array}$ & $\begin{array}{l}468 \text { (56.3\%) } \\
364(43.8 \%)\end{array}$ & $p<0.85$ \\
\hline $\begin{array}{l}\text { Procedure type } \\
\text { Bilateral } \\
\text { Left } \\
\text { Right }\end{array}$ & $\begin{array}{l}10(5.6 \%) \\
94(53.1 \%) \\
73(41.2 \%)\end{array}$ & $\begin{array}{c}13(1.6 \%) \\
447(53.7 \%) \\
372(44.7 \%)\end{array}$ & $p<0.004$ \\
\hline $\begin{array}{l}\text { Race } \\
\text { White } \\
\text { Black/African } \\
\text { American } \\
\text { Hispanic/Latino } \\
\text { Other }\end{array}$ & $\begin{array}{c}78(44.1 \%) \\
57(32.2 \%) \\
30(16.9 \%) \\
12(6.8 \%)\end{array}$ & $\begin{array}{l}418(50.2 \%) \\
224(26.9 \%) \\
133(16.0 \%) \\
57(6.9 \%)\end{array}$ & $p<0.677$ \\
\hline $\begin{array}{l}\text { Encounter type } \\
\text { Inpatient } \\
\text { Outpatient }\end{array}$ & $\begin{array}{c}144 \text { (8I.4\%) } \\
33(18.6 \%)\end{array}$ & $\begin{array}{l}531(63.8 \%) \\
301(36.2 \%)\end{array}$ & $p<0.001$ \\
\hline $\begin{array}{l}\text { Central venous } \\
\text { catheter use } \\
\text { Yes } \\
\text { No }\end{array}$ & $\begin{array}{c}38(26.8 \%) \\
139(78.5 \%)\end{array}$ & $\begin{array}{l}104(12.5 \%) \\
728(87.5 \%)\end{array}$ & $p<0.002$ \\
\hline $\begin{array}{l}\text { PICC use } \\
\text { Yes } \\
\text { No }\end{array}$ & $\begin{array}{c}31 \text { (17.5\%) } \\
146(82.5 \%)\end{array}$ & $\begin{array}{c}56(6.7 \%) \\
776(93.3 \%)\end{array}$ & $p<0.001$ \\
\hline $\begin{array}{l}\text { Pacemaker/CID } \\
\text { Yes } \\
\text { No }\end{array}$ & $\begin{array}{c}20(11.3 \%) \\
157(88.7 \%)\end{array}$ & $\begin{array}{c}68(8.2 \%) \\
764(91.8 \%)\end{array}$ & $p<0.181$ \\
\hline $\begin{array}{l}\text { Hypertension } \\
\text { Yes } \\
\text { No }\end{array}$ & $\begin{array}{c}120(67.8 \%) \\
51(28.8 \%)\end{array}$ & $\begin{array}{l}475(57.1 \%) \\
357(42.9 \%)\end{array}$ & $p<0.001$ \\
\hline $\begin{array}{l}\text { Diabetes mellitus } \\
\text { Yes } \\
\text { No }\end{array}$ & $\begin{array}{l}59(33.3 \%) \\
118(66.7 \%)\end{array}$ & $\begin{array}{l}265(31.9 \%) \\
567(68.1 \%)\end{array}$ & $p<0.701$ \\
\hline $\begin{array}{l}\text { Heart Disease } \\
\text { Yes } \\
\text { No }\end{array}$ & $\begin{array}{l}82(46.3 \%) \\
95(53.7 \%)\end{array}$ & $\begin{array}{l}347(41.7 \%) \\
485 \text { (58.3\%) }\end{array}$ & $p<0.259$ \\
\hline $\begin{array}{l}\text { Thrombophilia } \\
\text { Yes } \\
\text { No }\end{array}$ & $\begin{array}{c}27(15.3 \%) \\
150(84.7 \%)\end{array}$ & $\begin{array}{c}94(11.3 \%) \\
738(88.7 \%)\end{array}$ & $p<0.307$ \\
\hline $\begin{array}{l}\text { Malignancy } \\
\text { Yes } \\
\text { No }\end{array}$ & $\begin{array}{c}48(27.1 \%) \\
132(74.6 \%)\end{array}$ & $\begin{array}{l}159(19.1 \%) \\
673(80.9 \%)\end{array}$ & $p<0.053$ \\
\hline
\end{tabular}

(Continued)
Table I (Continued).

\begin{tabular}{|l|c|c|c|}
\hline $\begin{array}{l}\text { Characteristicl } \\
\text { Comorbidity }\end{array}$ & DVT, n (\%) & $\begin{array}{c}\text { No DVT, } \\
\text { n (\%) }\end{array}$ & Significance \\
\hline $\begin{array}{l}\text { Pulmonary disease } \\
\text { Yes }\end{array}$ & $61(34.5 \%)$ & $274(32.9 \%)$ & $p<0.695$ \\
No & $116(65.5 \%)$ & $558(67.1 \%)$ & \\
\hline $\begin{array}{l}\text { Chronic kidney } \\
\text { disease }\end{array}$ & & & \\
Yes & $61(34.5 \%)$ & $173(20.8 \%)$ & $p<0.001$ \\
No & $116(65.5 \%)$ & $659(79.2 \%)$ & \\
\hline
\end{tabular}

$d f$ 19, $p=0.401$; Pearson deviance $\chi^{2}=21.424 d f 19, \mathrm{p}=0.314$ ). Finally, Pearson residuals for our observed and predicted frequencies were less than the absolute value of two, indicating that our covariate patterns fit well by this model. As a result of our regression, CVC use, PICC-line use, malignancy, hypertension, and CKD were considered independent predictors ofUEDVT based on aORs and 95\% CIs (Table 2).

\section{Morbidity and Mortality}

There were no morbidities (pulmonary embolism, stroke, or right heart failure) in patients diagnosed with UEDVT. Patient mortality over a 365-day follow-up occurred in 91 (10.9\%) patients without diagnoses of UEDVT compared to $30(17.05 \%)$ patients with the diagnosis (OR 1.729, 95\% CI 1.108-2.697; $p<0.015)$. Mean time from index venous duplex study to death in patients diagnosed with UEDVT was $46.70 \pm 56.46$ days and $41 \pm 38.87$ days for those without UEDVT ( $\mathrm{p}=0.491)$. In sum, $21 \quad(70 \%)$ of the total mortalities with UEDVT were found to have

Table 2 Comorbidities significantly correlated with UEDVT diagnosis

\begin{tabular}{|l|c|c|c|c|c|}
\hline $\begin{array}{l}\text { Significantly } \\
\text { correlated } \\
\text { factor }\end{array}$ & $\boldsymbol{R}^{\mathbf{a}}$ & $\mathbf{O R}^{\mathbf{b}}$ & $\mathbf{C l}^{\mathbf{c}}$ & $\mathbf{a O R}$ & $\mathbf{a C l}^{\mathbf{c}}$ \\
\hline CVC use & 0.098 & 1.914 & $1.266-2.894$ & 1.770 & $1.150-2.725$ \\
PICC line & 0.146 & 2.942 & $1.833-4.722$ & 3.254 & $1.997-5.304$ \\
Hypertension & 0.109 & 1.857 & $1.304-2.643$ & 1.641 & $1.136-2.369$ \\
CKD & 0.123 & 2.003 & $1.408-2.849$ & 1.743 & $1.201-2.531$ \\
Malignancy & 0.095 & 1.443 & $0.987-2.110$ & 1.475 & $0.994-2.190$ \\
\hline
\end{tabular}

Notes: a Pearson's Correlation Coefficient, $p<0.005$; ${ }^{b} p<0.05$.

Abbreviations: CVC, central venous catheter; PICC, peripherally implanted central catheter; CKD, chronic kidney disease. 


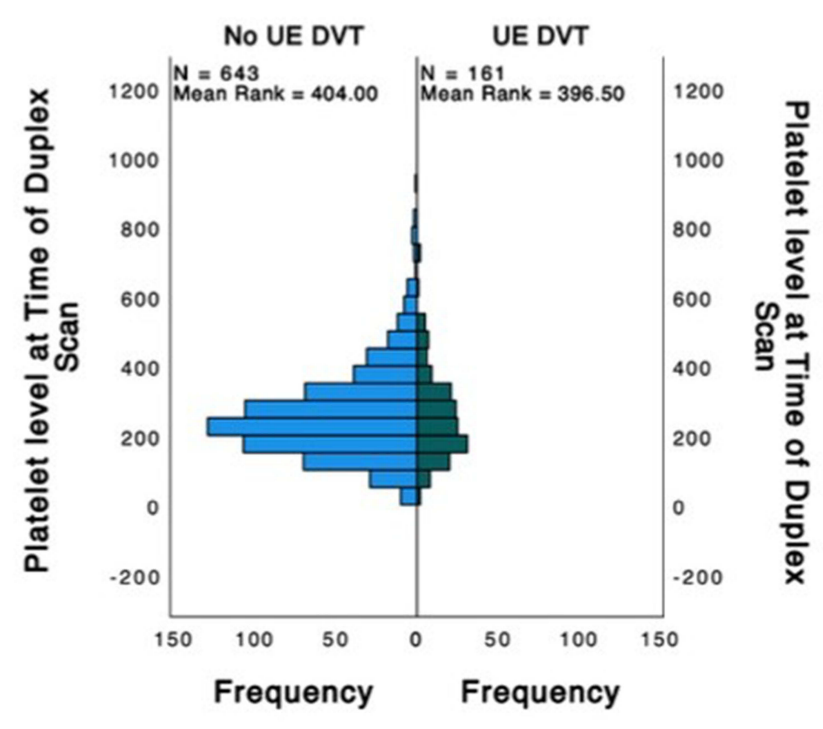

Figure I Platelet-distribution comparison between UEDVT and no-UEDVT groups. Distribution of platelet levels for 643 patients without UEDVT diagnosis seen on left, with mean platelet measurement 26I.69 132.64 , median 242, minimum I0, and maximum 927. Distribution of platelet levels for 161 patients with UEDVT seen on right, with mean platelet measurement $256.76 \pm$ | 28.476, median 234, minimum 26, and maximum 733 .

occurred $<30$ days after index venous Doppler scan. When evaluating independent predictors, of patients diagnosed with UEDVT who died within 30 days, six (28.6\%) had CVCs, five (23.8\%) PICC lines, ten (47.6\%) hypertension, five (23.8\%) malignancy, and eight (38.1\%) CKD. The 365 -mortality rates for patients were similar to the respective presence of independent risk factors: nine $(30 \%)$ had CVCs, six (20\%) PICC lines, 16 (53.3\%) hypertension, (30\%) malignancy, and ten (33.33\%) CKD.

None of the mortalities was documented as being directly attributed to UEDVT, but rather to other indirect causes. Causes of death included acute respiratory failure (23), cardiopulmonary arrest (51), sepsis/septic shock (20), or other causes (15).

\section{Discussion}

\section{Principal Findings}

This study succeeded in analyzing data of a large patient cohort at a level I trauma center to identify independent predictors of UEDVTs. It provides new information on the prevalence of UEDVT in the outpatient population compared to the inpatient population. This study was comprehensive in that it evaluated demographic factors, comorbidities, and hematological indices, as well as usage of invasive venous catheters. We confirmed that CVC use, PICC use, hypertension, malignancy, and CKD function were independent risk factors of UEDVT. Our analysis of hematological indices, namely platelet levels, demonstrated no relationship to increased UEDVT risk.

Venous thromboembolism poses a notable detriment to both public health and the health outcomes of hospitalized patients. An extensive and comprehensive body of evidence can be found in the literature on risk factors, morbidity, and mortality in cases of LEDVT; however, less is known about risk factors of UEDVT. ${ }^{1,2}$ Venous duplex ultrasound is both highly sensitive (91\%) and specific (93\%) when used for the identification of suspected UEDVTs. ${ }^{8}$ The American Institute of Ultrasound in Medicine has proposed 16 core indications for peripheral venous ultrasound, including evaluation for suspected DVT. ${ }^{9}$ In our study, a majority of indications to obtain a unilateral or bilateral upper-extremity venous Doppler ultrasound were upper-extremity pain, upper-extremity swelling, or upper-extremity pain and swelling combined.

\section{Risk Factors of UEDVT Diagnosis}

Several studies have investigated the various risk factors associated with UEDVTs and their clinical outcomes. In one retrospective study, 373 patients at risk of UEDVT with or without typical symptoms and signs were screened by venous duplex ultrasound, with 94 (25\%) ultimately diagnosed with acute UEDVT. ${ }^{3}$ The most common risk factors associated with UEDVT found in this study population were indwelling CVCs (93\%), with almost half of these patients with PICC placements (44\%), immobilization $>3$ days $(71 \%)$, and cancer $(49 \%)$, consistent with previous studies. ${ }^{3}$ All patients who died within 1 month after a UEDVT diagnosis had cancer and a CVC. ${ }^{3}$ We had similar findings, where $38(21.5 \%)$ of all UEDVTs included use of CVCs. In addition, we found central venous lines to be an independent risk factor of UEDVT: 38 (26.8\%) versus 139 (78.5\%); aOR 1.770 95\% CI 1.150-2.725). Therefore, it is suggested that patients who are at high risk of UEDVT whose current diagnoses indicate the need for CVCs be screened. Additionally, clinicians should follow guidelines on appropriate and minimal catheter use in high-risk patients. ${ }^{25}$

A case-control study of 239 patients demonstrated that the risk of a CVC associated with UEDVT was related to vessel size, catheter size, catheter positioning, and the type of catheter used, with the PICC being the most thrombogenic. ${ }^{10}$ Central venous lines disrupt the endothelial lining of large veins and alter hemodynamic flow, due to their presence in the venous lumen, creating stasis. 
These factors create a hypercoagulable state, as they disrupt the harmonious flow of Virchow's triad, increasing the chances of thrombus formation. We also found the use of PICC lines to be the most thrombogenic as an independent risk factor, with higher incidence of patients with UEDVTs compared to those without PICC lines. The higher incidence of UEDVTs in patients with PICC lines, as opposed to central venous lines, may be explained by the fact that PICC lines traverse a longer route through the venous system and have a higher catheter:vein-diameter ratio than central lines, all increasing hypercoagulability. ${ }^{11}$ What was interesting to see was that while PICC lines and CVCs posed significantly increased risk of developing UEDVT, patients with implanted pacemakers or cardiac implantable electronic devices did not have an increased risk of developing UEDVT $(p=0.181)$. This could be explained by the varying approaches to implanting such devices (either endovascularly or surgically) and the fact that a shorter vein is disturbed with endovascular placement compared to central lines or PICC lines, decreasing the risk of thrombus development. ${ }^{11}$

The influence of hypertension on the development of DVTs in general has been debated in previous studies, where some studies suggest statistical significance and others proclaim to show no statistically significant association. ${ }^{12-17}$ As our study has shown that the diagnosis of hypertension is an independent risk factor of the occurrence of UEDVT, potential theories on the mechanism of pathogenesis arise. For instance, just as chronic hypertension creates arterial-wall remodeling and increased inflammatory markers, some studies have shown that longstanding hypertension is associated with venous-wall remodeling, especially with regard to venous valves. ${ }^{17}$ Vascular-wall remodeling plays a role in the disruption of the normal microscopic anatomical structure, allowing for an increased predisposition for thrombus formation. ${ }^{17}$ In contrast, patients with heart disease or diabetes mellitus did not present with a significantly increased risk for developing UEDVT (Table 2). Just as with hypertension, both diabetes mellitus and heart disease are defined by varying degrees of severity, management, and control. Therefore, although we did not find any correlation between heart disease or diabetes mellitus with diagnosis of UEDVT in our patient population, further studies in the area may be needed. Additionally, further prospective studies investigating outcomes with respect to stage, duration, and control of hypertension would help further elucidate the risk.
CKD was also an independent predictor of UEDVT compared to those who had CKD. CKD is a known risk factor of LEDVT, especially in patients with end-stage renal disease, those who are receiving hemodialysis, or those with a history of renal transplant. Some studies have discussed the potential role of increased fibrinogen levels and inflammatory markers, especially in nephrotic syndromes, in the development of DVT. ${ }^{18,19}$ In addition, patients with CKD have been shown to have decreased endogenous anticoagulation proteins secondary to proteinuria, endothelial cell dysfunction, enhanced platelet activation and aggregation, and enhanced activation of the coagulation system. ${ }^{19}$

It is interesting to note that a medical history of malignancy was associated with statistically significant incidence compared to no medical history of same. This parallels the findings in past studies, where the inflammatory environment created by malignant tumors promoted a hypercoagulable state, especially in more advanced and aggressive malignancies. ${ }^{3,4}$ Although our study cohort consisted of a heterogeneous pool of malignancies encompassing various disease sites and stages, and information on ongoing treatment plans or malignancies being active at the time of upper-extremity duplex scans not being consistently available, it still allows us to generalize risk in this population. As such, oncological patients presenting with upper-extremity pain and/or swelling should be considered with a high index of clinical suspicion for UEDVT.

The role of thrombophilia, defined as either congenital or acquired disorders of coagulation leading to hypercoagulable states, in the formation of UEDVT requires further elucidation. One case-control study where 150 patients with UEDVT were compared to 300 with LEDVT found that at least one thrombophilic disorder was found in $34 \%$ of all UEDVT patients. ${ }^{6}$ However, the overall prevalence of at least one hereditary or acquired thrombophilic disorder reported in patients with UEDVT was significantly less than that of patients with LEDVT. ${ }^{6}$ In our study, 27 (15.3\%) patients who had been previously diagnosed with thrombophilia developed acute UEDVT, while 150 $(84.7 \%)$ without a previous diagnosis of thrombophilia developed UEDVT, a difference that was nonsignificant $(p<0.307)$. This leads to consideration of a type II error in hypothesis testing, as our statistical power was not designed to study this risk factor. The documented thrombophilias in this study were heterogeneous, and thus further studies may be warranted to 
investigate correlation among types of thrombophilia, severity of the conditions, and incidence of UEDVTs.

Several studies have demonstrated the increased risk of LEDVTs due to reactive thrombocytosis during the recovery period in severely ill patients, especially after trauma, chemoprophylaxis, or coronary artery-bypass surgery. ${ }^{19-23}$ In contrast, our study revealed no relationship between platelet levels and diagnosis of UEDVT. A notable difference in our study was that platelet levels were measured at the time of upper-extremity Doppler ultrasound, whereas some of of previous literature analyzed platelet levels after ICU discharge. ${ }^{19}$ Therefore, longitudinal evaluation of the platelet levels of our patients may be warranted, in order to demonstrate the reactive thrombocytosis hypothesis at other times during their illness. In addition, since only platelet levels were readily available in this retrospective study, further studies can be conducted in order to analyze other markers of hypercoagulability, such as bleeding time, PT, PTT, and INR levels, which may provide more information to formulate any correlation between platelets and/or coagulation factors and UEDVTs.

\section{Diagnosis of UEDVT and Mortality}

In this study, $30(17.05 \%)$ patients with UEDVT died within 365 days of their index venous duplex scan, with $21(70 \%)$ of those occurring within 30 days of the index scan. The small samples in both mortality groups may have affected further analysis. What is more, many of the deaths were secondary to causes other than typical complications of an UEDVT, and cause of death was not consistently documented in all cases to accurately ascertain the existence of any correlation with UEDVT. In addition, no morbidity was documented as secondary to UEDVT in our study. Based on the literature, the most common cause of mortality is pulmonary embolism, although this is more commonly associated with LEDVT. ${ }^{24}$ In one retrospective study, 9\% of 373 patients with UEDVT studied presented with pulmonary embolism, but no direct correlation was found between pulmonary embolism and mortality. ${ }^{3}$

This study was performed at a single center, and thus the results are limited by the patient population of the center. The information provided by this study will be valuable in identifying patients at risk of UEDVT, modifying their subsequent management, and mitigating the occurrence of UEDVT. Management of UEDVT relies on the cornerstone treatment of anticoagulation, with early treatment aimed at obtaining early venous recanalization and attempting to restore vein patency. Management is also largely based on the etiology of UEDVT, and thus awareness of identifying patients with significant risk factors will be beneficial in creating more accurate and effective treatment plans for each patient. For example, low-molecular weight heparin is the preferred method of anticoagulation in malignancyassociated UEDVT, with therapy continuing for at least 3 months. ${ }^{26}$ In another example, treatment for CVC-associated UEDVT is removal of the offending catheter if it is no longer needed orworking with concurrent therapeutic anticoagulation. Anticoagulation should be administered for as long as the CVC remains if it is necessary, and should continue for 3 months after its removal. ${ }^{26}$

Further study is warranted at a multicenter level to further analyze the impact on various patient populations, where the increased number of patients in a cohort can increase the study power. Weaknesses include the retrospective nature of this study, which limited our understanding of causes of mortality, severity of disease comorbidities, and precise medical history of active malignancy stage and treatment. What is more, all cases with a diagnosis of UEDVT were compared to cases where upper-extremity duplex scans had been performed secondary to symptomatic indications. This may point to the value of performing a future prospective caserandomized control trial. In addition, challenges associated with ICD coding and lack of documented information on duration of patient immobilization and anticoagulation status for outpatients were limiting factors in this study. Further studies are warranted to ascertain the relationship of thrombophilia to the development of UEDVT.

\section{Conclusion}

Overall, our study comprehensively evaluated risk factors and found that CVCs, PICC lines, diagnosis of hypertension, CKD, and malignancy were independent predictors for development of UEDVT. Platelet level and presence of thrombophilia were not found to be risk factors. This information will aid health-care providers in more accurately identifying patients at risk of UEDVT and guide subsequent management.

\section{Abbreviations}

UEDVTh, upper-extremity deep-vein thrombosis; LEDVT, lower-extremity DVT; PICC, peripherally inserted central catheter; CKD, chronic kidney disease; $\mathrm{CVC}$, central venous catheter. 


\section{Disclosure}

The authors reported no conflicts of interest for this work.

\section{References}

1. Grigorian A, Nahmias JT Upper Extremity Deep Venous Thrombosis. StatPearls [Internet]. Treasure Island (FL): StatPearls Publishing; 2020. Available from: https://www.ncbi.nlm.nih.gov/books/ NBK482420/. Accessed June 3, 2021.

2. Ploton G, Pistorius M, Raimbeau A, et al. A STROBE cohort study of 755 deep and superficial upper-extremity vein thrombosis. Medicine. 2020;99(6):e18996. doi:10.1097/MD.0000000000018996

3. Lee JA, Zierler BK, Zierler RE. The risk factors and clinical outcomes of upper extremity deep vein thrombosis. Vasc Endovascular Surg. 2012;46(2):139-144. doi:10.1177/1538574411432145

4. Martinelli I, Battaglioli T, Bucciarelli P, Passamonti SM, Mannucci PM. Risk factors and recurrence rate of primary deep vein thrombosis of the upper extremities. Circulation. 2004;110 (5):566-570. doi:10.1161/01.CIR.0000137123.55051.9B

5. McLendon K, Goyal A, Bansal P, et al. Deep Venous Thrombosis Risk Factors. StatPearls [Internet]. Treasure Island (FL): StatPearls Publishing; 2020. Available from: https://www.ncbi.nlm.nih.gov/ books/NBK470215/. Accessed June 3, 2021.

6. Linnemann B, Meister F, Schwonberg J, et al. Hereditary and acquired thrombophilia in patients with upper extremity deep-vein thrombosis. Results from the MAISTHRO registry. Thromb Haemost. 2008;100(3):440-446. doi:10.1160/TH08-03-0196

7. Di Nisio M, Van Sluis GL, Bossuyt PM, Büller HR, Porreca E, Rutjes AW. Accuracy of diagnostic tests for clinically suspected upper extremity deep vein thrombosis: a systematic review. $J$ Thromb Haemost. 2010;8(4):684-692. doi:10.1111/j.15387836.2010.03771.x

8. Practice AIUM. Parameter for the Performance of a Peripheral Venous Ultrasound Examination. J Ultrasound Med. 2020;39(5): E49-E56.

9. Winters JP, Callas PW, Cushman M, Repp AB, Zakai NA. Central venous catheters and upper extremity deep vein thrombosis in medical inpatients: the Medical Inpatients and Thrombosis (MITH) Study. J Thromb Haemost. 2015;13(12):2155-2160. doi:10.1111/jth.13131

10. Bonizzoli M, Batacchi S, Cianchi G, et al. Peripherally inserted central venous catheters and central venous catheters related thrombosis in post-critical patients. Intensive Care Med. 2011;37 (2):284-289. doi:10.1007/s00134-010-2043-x

11. Alanazi OA, El-Fetoh NMA, Mohammed NA, et al. Deep Venous Thrombosis among hypertensive patients in King Abdulaziz University (KAU) Hospital, Jeddah, Kingdom of Saudi Arabia. Electron Physician. 2017;9(10):5472-5477. doi:10.19082/5472

12. Huang L, Li J, Jiang Y. Association between hypertension and deep vein thrombosis after orthopedic surgery: a meta-analysis. Eur J Med Res. 2016;21(1):13. doi:10.1186/s40001-016-0207-z
13. Ageno W, Becattini C, Brighton T, Selby R, Kamphuisen PW. Cardiovascular risk factors and venous thromboembolism. Circulation. 2008;117(1):93-102. doi:10.1161/CIRCULATIONAHA. 107.709204

14. Song EK, Kim JK, Lee KB, Seon JK. Deep vein thrombosis after total knee replacement: incidence and correlation with clinical risk factors. J Korean Knee Soc. 1998;10(1):18.

15. Wang CJ, Wang JW, Chen LM, Chen HS, Yang BY, Cheng SM. Deep vein thrombosis after total knee arthroplasty. $J$ Formos Med Assoc. 2000;99(11):848-853.

16. Mazzoccoli G, Grilli M, Ferrandino F, et al. Arterial endothelial dysfunction and idiopathic deep venous thrombosis. J Biol Regul Homeost Agents. 2011;25(4):565-573.

17. Wattanakit K, Cushman M. Chronic kidney disease and venous thromboembolism: epidemiology and mechanisms. Curr Opin Pulm Med. 2009;15(5):408-412. doi:10.1097/MCP.0b013e32832ee371

18. Wattanakit K, Cushman M, Stehman-Breen C, Heckbert SR, Folsom AR. Chronic kidney disease increases risk for venous thromboembolism. J Am Soc Nephrol. 2008;19(1):135-140. doi:10.1681/ASN.2007030308

19. Ho KM, Yip CB, Duff O. Reactive thrombocytosis and risk of subsequent venous thromboembolism: a cohort study. J Thromb Haemost. 2012;10(9):1768-1774. doi:10.1111/j.1538-7836. 2012.04846.x

20. Salim A, Hadjizacharia P, DuBose J, et al. What is the significance of thrombocytosis in patients with trauma? J Trauma. 2009;66 (5):1349-1354. doi:10.1097/TA.0b013e318191b8af

21. Kashuk JL, Moore EE, Johnson JL, et al. Progressive postinjury thrombocytosis is associated with thromboembolic complications. Surgery. 2010;148(4):667-675. doi:10.1016/j.surg.2010.07.013

22. Duff OC, Ho KM, Maybury SM. In vitro thrombotic tendency of reactive thrombocytosis in critically ill patients: a prospective case-control study. Anaesth Intensive Care. 2012;40(3):472-478. doi:10.1177/0310057X1204000313

23. Schmuziger M, Christenson JT, Maurice J, Simonet F, Velebit V. Reactive thrombocytosis after coronary bypass surgery. An important risk factor. Eur $J$ Cardiothorac Surg. 1995;9(7):393-398. doi:10.1016/S1010-7940(05)80172-1

24. Wilbur J, Shian B. Diagnosis of deep venous thrombosis and pulmonary embolism. Am Fam Physician. 2012;86(10):913-919.

25. O'Grady NP, Alexander M, Burns LA, et al. Summary of recommendations: guidelines for the Prevention of Intravascular Catheter-related Infections. Clin Infect Dis. 2011;52(9):1087-1099. doi:10.1093/cid/cir138

26. Kearon C, Akl EA, Ornelas J, et al. Antithrombotic Therapy for VTE Disease: CHEST Guideline and Expert Panel Report [published correction appears in Chest. Chest. 2016;149(2):315-352. doi:10.1016/j. chest.2015.11.026
International Journal of General Medicine

\section{Publish your work in this journal}

The International Journal of General Medicine is an international, peer-reviewed open-access journal that focuses on general and internal medicine, pathogenesis, epidemiology, diagnosis, monitoring and treatment protocols. The journal is characterized by the rapid reporting of reviews, original research and clinical studies across all disease areas. The manuscript management system is completely online and includes a very quick and fair peer-review system, which is all easy to use. Visit http://www.dovepress.com/ testimonials.php to read real quotes from published authors. 